\title{
Supplement materials of
}

\section{A data-driven topsoil $\delta 13 \mathrm{C}$ dataset and the drivers of spatial}

\author{
variability across the Tibetan Plateau
}

\section{Yunsen Lai ${ }^{1}$, Shaoda $\mathrm{Li}^{1}{ }^{1,}$ *, Yuehong Shi ${ }^{1}$, Xinrui Luo ${ }^{1}$, Liang Liu ${ }^{1}$, Peng Yu ${ }^{1}$,}

Guo Chen ${ }^{2}$, Longxi Cao ${ }^{2}$, Chunju Cai ${ }^{3}$, Jian Sun ${ }^{4}$, Shaohui Chen ${ }^{5}$, Houyuan

$\mathrm{Lu}^{6,7,8}$, Xuanlong Ma ${ }^{9}$, Xiaolu Tang ${ }^{2,10, *}$

${ }^{1}$ College of Earth Science, Chengdu University of Technology, Chengdu 610059, Chengdu, China

${ }^{2}$ College of Ecology and Environment, Chengdu University of Technology, Chengdu 610059, Chengdu, China

${ }^{3}$ Key Laboratory of Bamboo and Rattan Science and Technology of National Forestry and Grassland Administration, International Centre for Bamboo and Rattan, Beijing 100102, China

${ }^{4}$ Key Laboratory of Alpine Ecology, Institute of Tibetan Plateau Research, Chinese Academy of Sciences, Beijing 100101, China

${ }^{5}$ Key Laboratory of Water Cycle and Related Land Surface Processes, Institute of Geographic Sciences and Natural Resources Research, Chinese Academy of Sciences, Beijing, 100101, China

${ }^{6}$ Center for Excellence in Tibetan Plateau Earth Science, Chinese Academy of Sciences, 100101, Beijing, China

${ }^{7}$ University of Chinese Academy of Sciences, 100049, Beijing, China

${ }^{8}$ Key Laboratory of Cenozoic Geology and Environment, Institute of Geology and Geophysics, Chinese Academy of Sciences, 100029, Beijing, China

${ }^{9}$ College of Earth and Environmental Sciences, Lanzhou University, Lanzhou, 730000, China

${ }^{10}$ State Environmental Protection Key Laboratory of Synergetic Control and Joint Remediation for Soil \& Water Pollution, Chengdu University of Technology, Chengdu 610059, Chengdu, China

*Shaoda Li, corresponding author at College of Earth Science, Chengdu University of Technology, Chengdu, China. Email: 1661348392@qq.com.

${ }^{*}$ Xiaolu Tang, corresponding author at College of Ecology and Environment, Chengdu University of Technology, Chengdu, China. Email: 1xtt2010@163.com. 


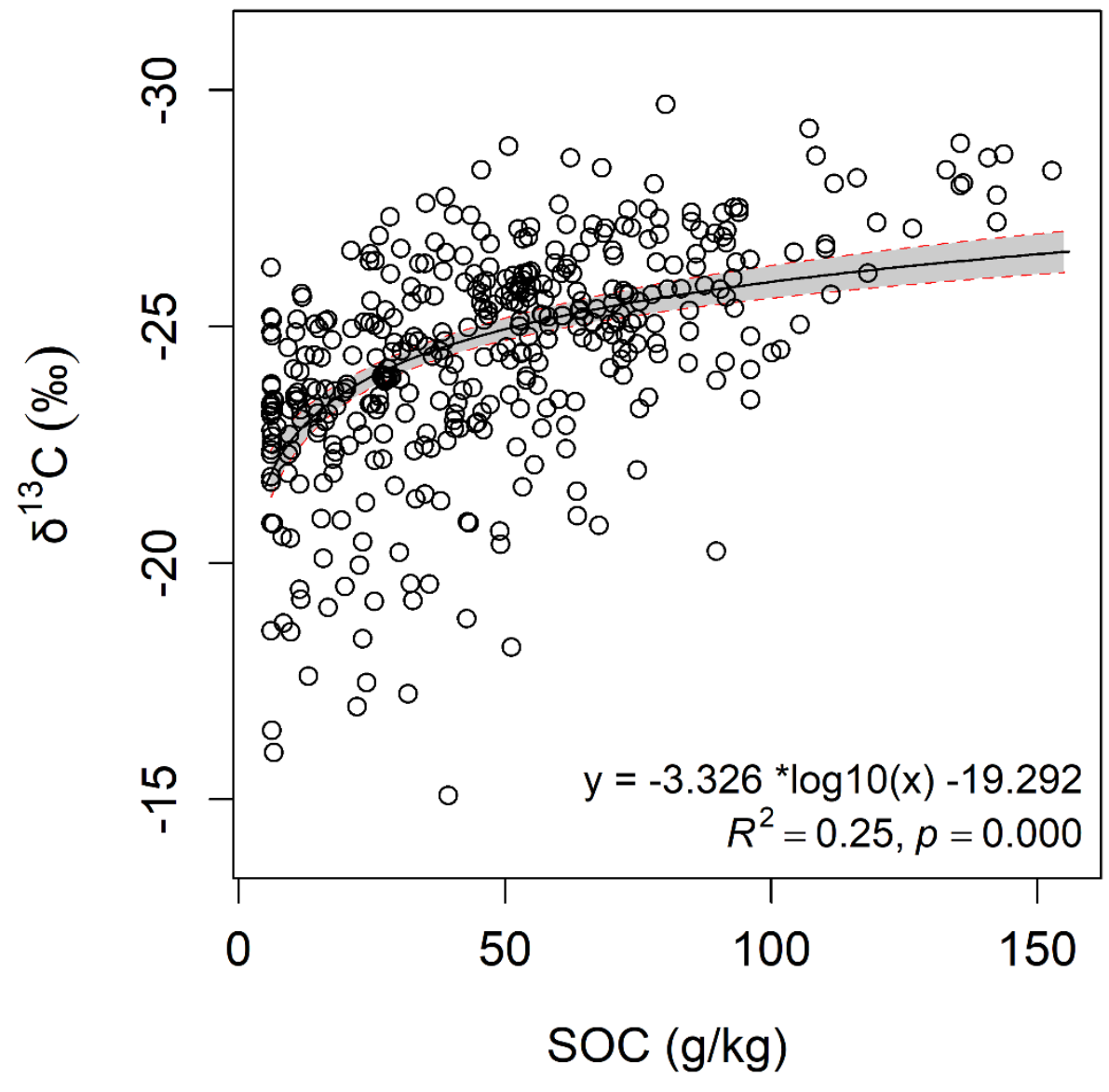

Figure S1. Relationships of soil $\delta^{13} \mathrm{C}$ with SOC content in 0-5 c 
Importance value of variables
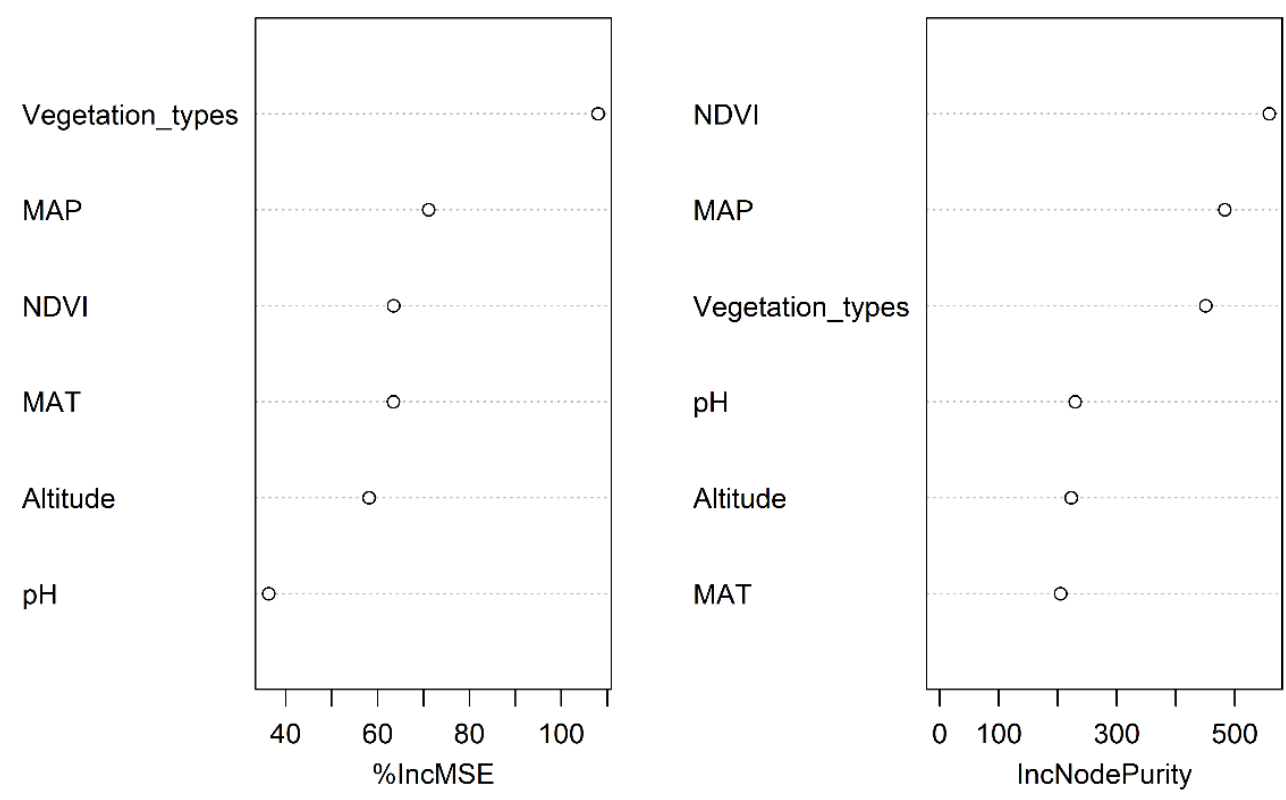

36 Figure S2. Importance of variables, denoted by percentage increase of mean-squared error (\%IncMSE) (left) and the increase in node purity (IncNodePurity) (right), for topsoil $\delta^{13} \mathrm{C}$ estimation RF model built from training dataset without considering plot location uncertainty. Vegetation_types, MAP, NDVI, MAT, Altitude, $\mathrm{pH}$, represent the ecosystem types, annual mean precipitation, normalized difference vegetation index, elevation, soil $\mathrm{pH}$, respectively. 


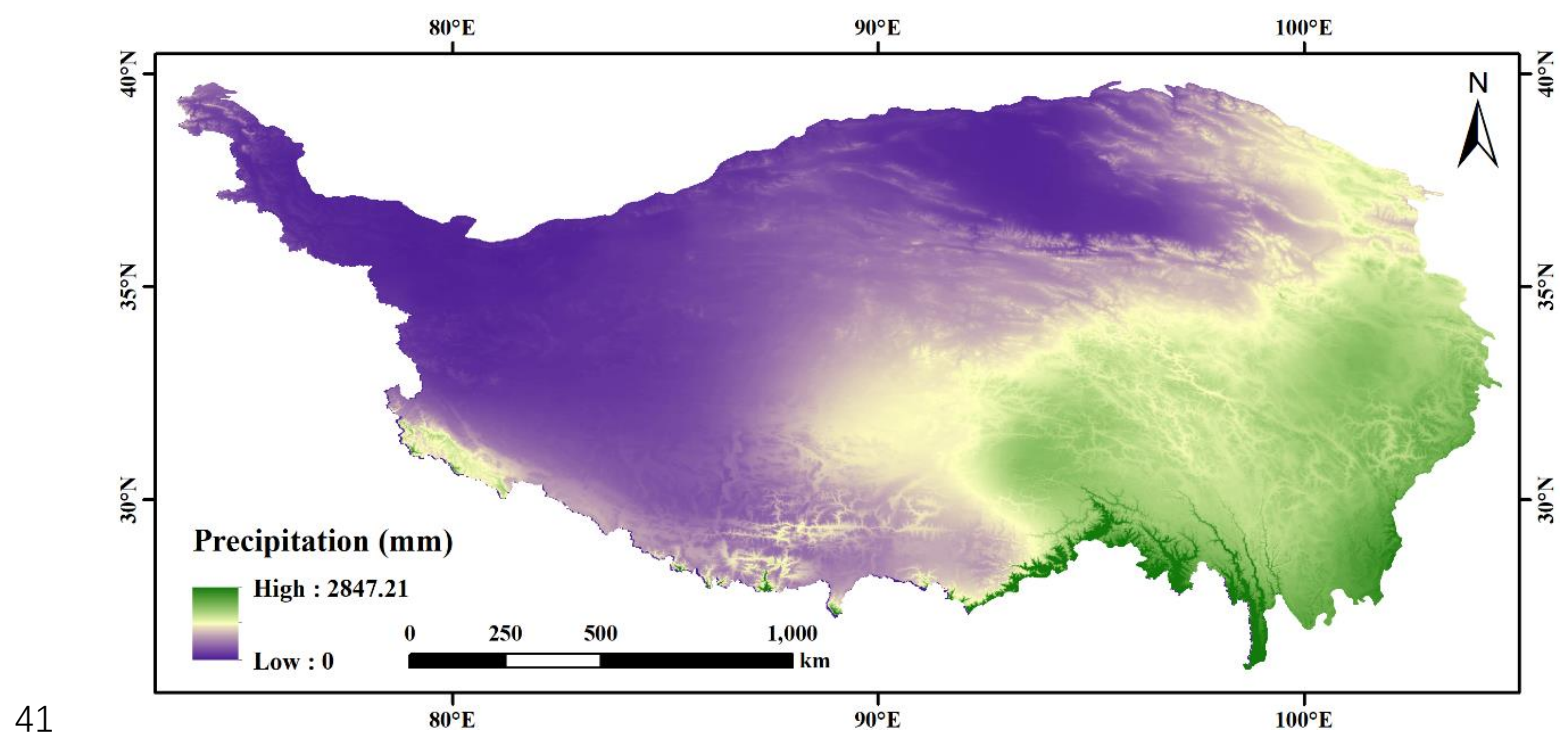

42 Figure S3. The distribution of precipitation during 2000-2010. 


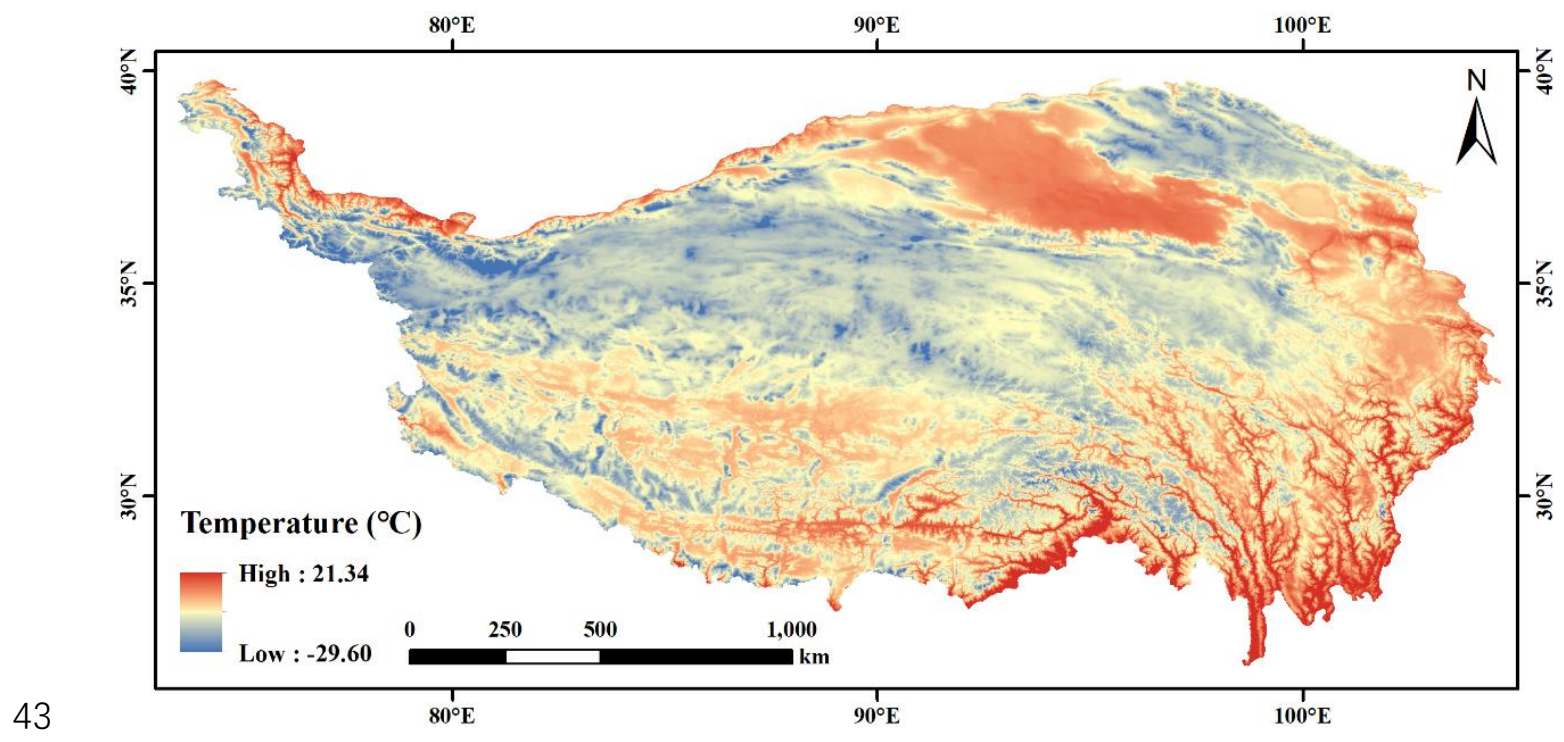

44 Figure S4. The distribution of temperature during 2000-2010. 


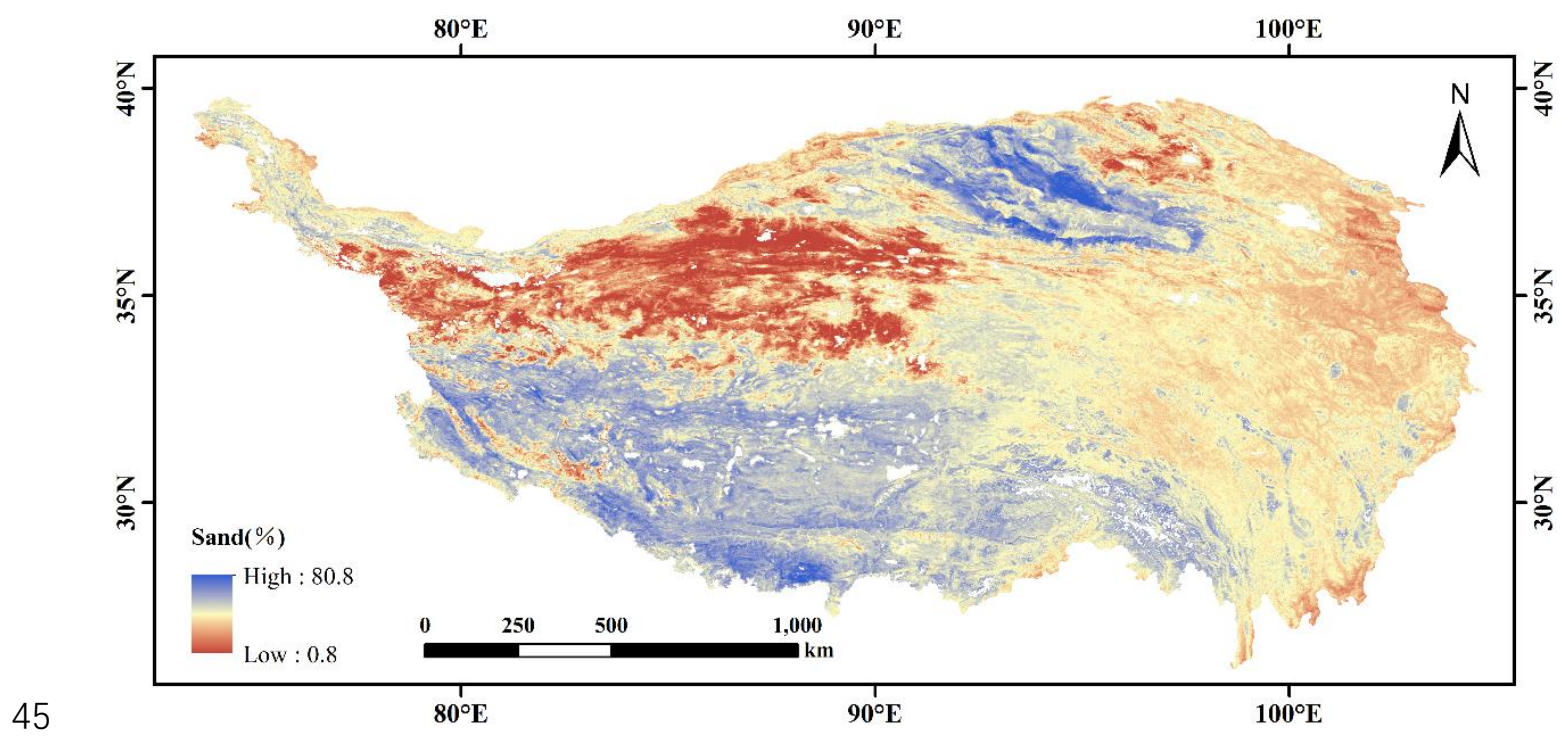

46 Figure S5. The distribution of soil sand content on the Tibetan Plateau. 


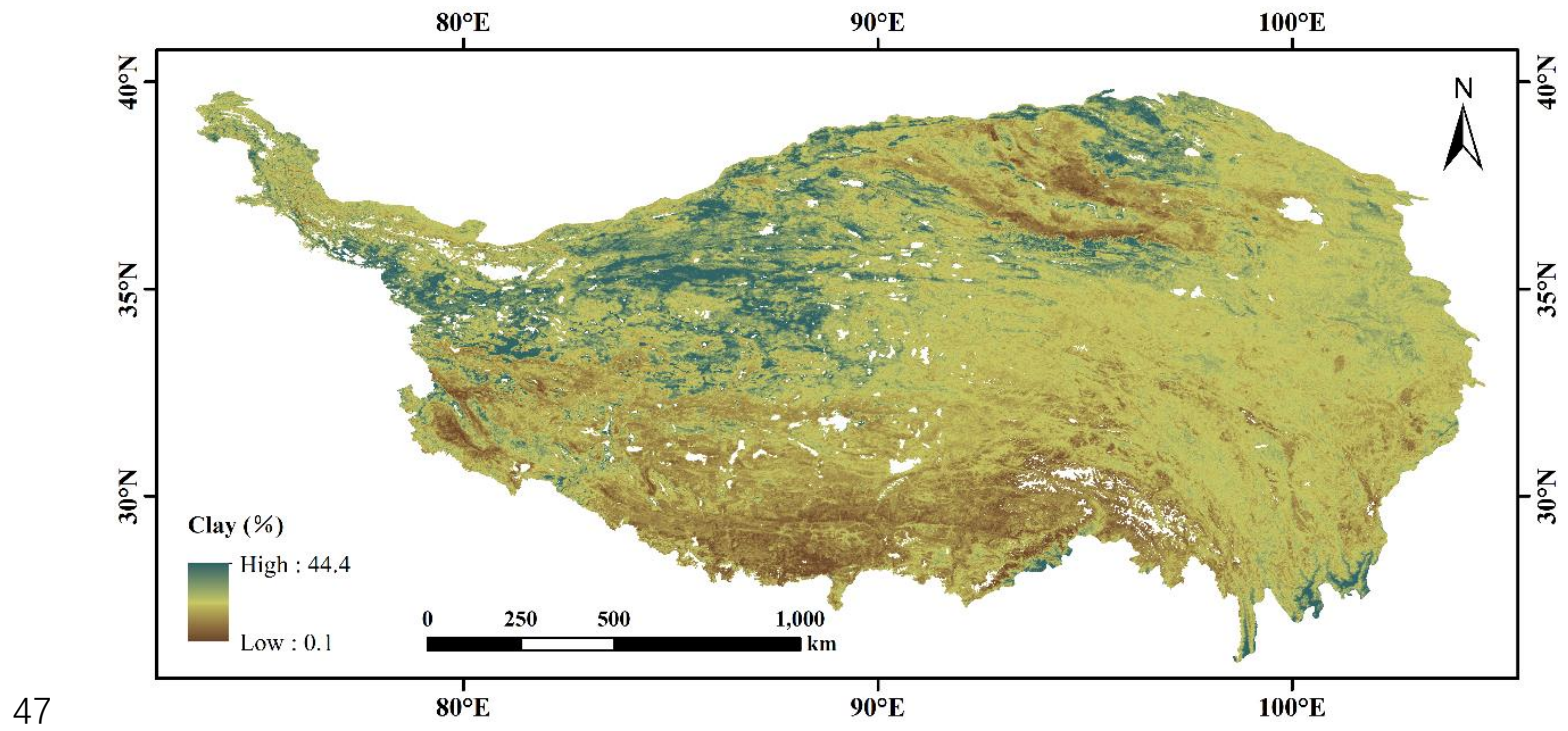

48 Figure S6. The distribution of soil clay content on the Tibetan Plateau. 


\begin{tabular}{lccccc}
\hline Ecosystem types & $\mathrm{N}$ & Mean & Standard & Minimum & Maximum \\
& & & Deviation & \\
\hline Forests & 103 & -26.3 & 1.60 & -29.7 & -18.8 \\
Shrublands & 59 & -24.3 & 2.00 & -28.8 & -18.2 \\
Grasslands & 218 & -23.9 & 1.84 & -27.6 & -18.5 \\
Deserts & 16 & -18.9 & 2.37 & -22.5 & -15.1 \\
\hline
\end{tabular}

$50 \mathrm{~N}$ are the number of soil samples. 